\title{
A parametric study of a tower controlled by a pendulum tuned mass damper: beam modelling
}

\author{
Gino B. Colherinhas ${ }^{1, *}$, Maura A. M. Shzu ${ }^{2}$, Suzana M. Avila ${ }^{2}$, and Marcus V. G. de Morais ${ }^{1}$ \\ ${ }^{1}$ Universidade de Brasília, Campus Universitário Darcy Ribeiro, Brasília, Distrito Federal, Brazil \\ ${ }^{2}$ Universidade de Brasília, Campus UnB Gama, Gama, Distrito Federal, Brazil
}

\begin{abstract}
This paper models a tower with a passive Pendulum Tuned Mass Damper (PTMD) with Finite Elements (FE) using the resources and capabilities of commercial software ANSYS. Although structural control of high and slender towers using PTMDs are widely studied in literature, it was not found yet studies modelling the PTMD with ANSYS. This FE model is called by a routine coded in MATLAB to find the relation between the mass, length, stiffness, and damping coefficient of the pendulum in function of the high vibration amplitudes at the top of the tower (defined as a beam element type). This parametric study of the dynamic behaviour of the PTMD + FE beam structural model is analysed and its results are compared to a genetic optimization developed in other researches to find the best pendulum parameters.
\end{abstract}

\section{Introduction}

Historically, the development and advancement of societies have been intimately tied to the members' ability to produce and manipulate structures and materials to fill theirs needs. The merge of construction techniques with numerical and experimental methods given rise to complex structures capable to assume a dynamic behaviour.

The modelling of high towers, in last decades, expressively increases. Due to their slenderness these structures can experience excessive vibrations caused by wind or seismic excitations. Some control devices, such as passive, active, or hybrid controllers, were widely studied.

Passive control consists in adding one or more devices to the main structure, which transfers part of its energy to control devices [1], for example: metallic dampers that dissipate their energy by plastic deformation and mass dampers which control structural responses transferring the main structure energy to an auxiliary mass.

The use of Tuned Mass Dampers (TMD) design for vibration suppression still increases. For example there are applications to a barge-type offshore floating wind turbine [2], the analytical formulae improvement for design optimization of Pendulum Tuned Mass Dampers (PTMD) [3], and experimental and numerical studies to examine seismic performance of the pendulum-type non-traditional tuned mass damper system (PNTTMD) to control structural vibrations [4].

\footnotetext{
*e-mail: ginobertollucci@ hotmail.com
} 
A PTMD is a pendulum-type passive control composed by a mass-spring-dashpot attached to the structure, aiming to reduce structural vibration responses [5]. A recently research modelled a simplified structure of a wind turbine [6, 7], using BEAM elements via Finite Element Method (FEM). Attached to the main structure, a PTMD minimizes peak responses by using a Genetic Algorithm optimization approach [8].

In this article an overview of previous studies is reached out by means of a parametric analysis of a tower controlled by a PTMD. Then can be understood the nuances between both analytical and numerical models by comparing their response maps, which contain the maximum peaks of the free vibration of the tower+PTMD in function of the pendulum length and the mass ratio between the pendulum and the main structure.

To plot the numerical response map a MATLAB routine integrated to the ANSYS APDL software was elaborated. For specific range of pendulum length and mass ratio, it was possible to call many instances of the ANSYS APDL, varying their inputs, creating new meshes, and performing modal analysis which founds the maximum response amplitude for each combination.

\section{Case study description}

A simplified model of a wind turbine which supports at its top the set of nacelle + blades can be modelled as a BEAM element-type. This BEAM structure with a tip mass attached to a PTMD is shown in Fig. 1.

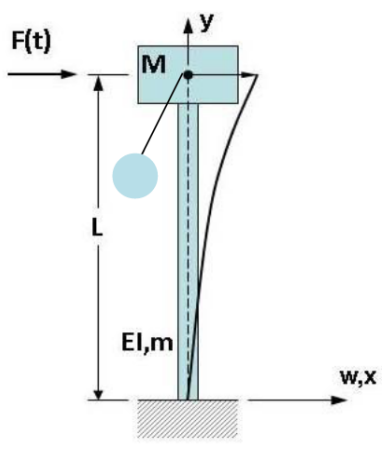

Figure 1. Description of a cantilever beam with a tip mass $m$ coupled by a pendulum TMD

The theoretical BEAM+PTMD approach is also considered by some authors $[9,10]$ assuming the dynamic contribution of wind rotor as compression-tension axial forces due to rotor angular velocity and gravitational force. The gravitational force causes parametric excitation on the blade, i.e., Mathieu's equation [11]. However, when considering small angular velocity of blades can be assumed a constant axial load [10]. This assumption is very advantageous for a preliminary analysis and it is used to validate numerical tools and approximate models presented in this paper.

Although the real structure is a system with infinite degrees-of-freedom, it can be modelled as discrete system with multi degrees-of-freedom (MDOF) in a Finite Element approach. The dynamic equilibrium equations of this MDOF system are:

$$
\mathbf{M} \ddot{y}(t)+\mathbf{C} \dot{y}(t)+\mathbf{K} y(t)=f(t)
$$


where $\mathbf{M}, \mathbf{C}$ and $\mathbf{K}$ are mass, damping and stiffness matrices, respectively. $\ddot{y}(t), \dot{y}(t)$ and $y(t)$ are acceleration, velocities and displacements vectors, respectively. $f(t)$ is the dynamic load vector. The solution of the free undamped vibration problem provides the natural frequencies and associate vibration modes of the system. Generally, structures, like the one studied in this work, usually vibrate predominantly on the fundamental mode.

\section{Finite element method applied to a BEAM+PTMD model}

The dynamic behaviour of this structure is studied through harmonic analysis. Aiming to approximate the MDOF to realistic models, a BEAM+PTMD is modelled using the ANSYS APDL $[6,7]$.

This work looks for a solution of the linear behaviour of a coupled pendulum system structure such as wind turbine towers or chimneys. The stiffness matrix of a bar subjected to axial load (gravity force), considering large displacements, can be obtained through Total Lagrangian formulation [12] as:

$$
\mathbf{K}=\mathbf{K}_{M}+\mathbf{K}_{G}
$$

where $\mathbf{K}_{M}$ is the material stiffness matrix and $\mathbf{K}_{G}$ is the geometric stiffness matrix. ANSYS take this effect into account incorporating the associated geometric matrix using PSTRES command.

Briefly, the tower is modelled as a BEAM element type with a MASS21 element, that defines the "nacelle+blade" mass, at its top. The mass-spring system with a PTMD is composed by MASS21, BEAM4 and COMBIN14 elements. The PTMD can be defined as EulerBernoulli BEAM4 element coupled with a MASS21 element at its end. A COMBIN14 element represents the system structural stiffness. Another COMBIN14 element models a threedimensional spring to describe the torsional stiffness that connects the tower to the pendulum via CP command. The structure is described using Timoshenko linear elements BEAM188 with a MASS21 element at the top. The pendulum is connected to the top of the structure via COMBIN14.

\section{Methodology}

This section describes the methodology used to create the response map that assists the parametric study of the tower. It consists in a routine developed in MATLAB, which calls instances of the ANSYS Mechanical APDL software to create the FEM BEAM+PTMD structure model finding its maximum response peak through a modal analysis. Figure 2 shows the flow chart which explains how the MATLAB routine works.

Firstly the main.m MATLAB file is initialized defining the pendulum length $x\left(L_{p}\right)$ and mass ratio $y(\mu)$ span. We write these and another constants inputs in a parameters . inp file, such as the tower mass $M_{t}$, pendulum damping and stiffness $C_{p}$ and $K_{p}$, respectively.

Each ANSYS APDL instance is called in a MATLAB code using 4 parallel intel processors, by allocating 16 GBytes in each OpenMP* thread in a private stack. Of course it is unnecessary use 16 GB for each private stack, but doing this it can always be avoided memory overflow. By setting 4 parallel processors it can saved also a lot of computational cost. The following code describes the way used to set these parameters:

system('SET KMP_STACKSIZE $=16 \mathrm{~g} \&$ "C: $\backslash$ ANSYS_directory $\backslash$ ANSYS181.exe" -mpi intelmpi -dyn -np 4 -b -i "C: \user_directory $\backslash$ model_BEAM.txt" -o "C: \user_directory \out_BEAM.txt"'); 


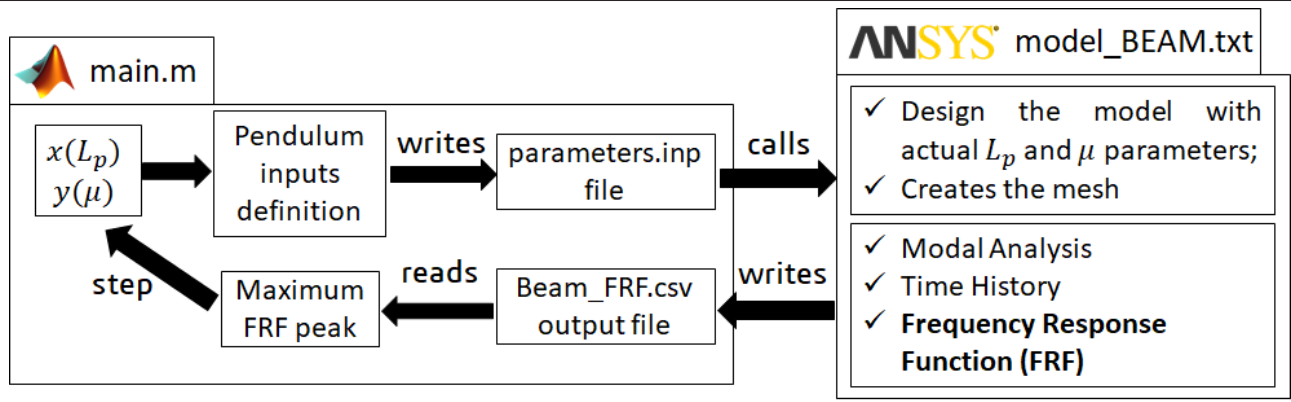

Figure 2. Methodology flow chart used to create the response map

After modal analysis, the last lines of the ANSYS APDL code writes the Frequency Response Function (FRF) in a BEAM_FRF.csv file. When the FEM analysis concludes, the main.m code reads the BEAM_FRF.CSV and determines its maximum peak. This peak is associated to a meshgrid of $L_{p}$ and $\mu$. The step proceeds repeating this processes until accomplishes the range.

\section{Results and parametric analysis}

The ranges used for $x\left(L_{p}\right)$ and $y(\mu)$ are the same as the restriction used in previous work of a genetic algorithm optimization [13], which are:

$$
\begin{array}{r}
0.50 \leq x \leq 10.00 \\
0.01 \leq y \leq 0.35
\end{array}
$$

To obtain the FEM BEAM response map of Fig. 3 the simulation spends almost 3 days to compute all FRF peaks, while the response map of the Analytical 2 Degrees of Freedom of Fig. 4 spends at most 10 minutes. For both simulations same inputs were used for the tower and PTMD.

Comparing both simulations it is possible to see the close resemblance between the FEM BEAM+PTMD and the Analytical 2DOF Tower+PTMD. In both cases it is notorious the geometric locus containing a valley. There are also visible some differences in regions far from the geometric locus. To see better the difference between both response maps, they are replaced in a XY log-log plan (Figs. 5 and 6).

We notice in Figs. 5 and 6 that these resemblances actually are pretty close only for mass ratios higher than $\mu>0.10$.

\section{Conclusions}

The parametric analysis can be very useful to assist PTMD designs. In this article it is compared both FEM BEAM+PTMD and Analytical 2DOF Tower+PTMD response maps. A methodology was developed to find maximum FRF peaks in function of the pendulum length $L_{p}$ and mass ratio $\mu$ spans. We can conclude that the use of Genetic Optimization in previous works [8] can find directly the geometric locus of the FEM BEAM+PTMD, but a complete overview of a response map shows us efficiently dynamic behaviour of this system. We can 


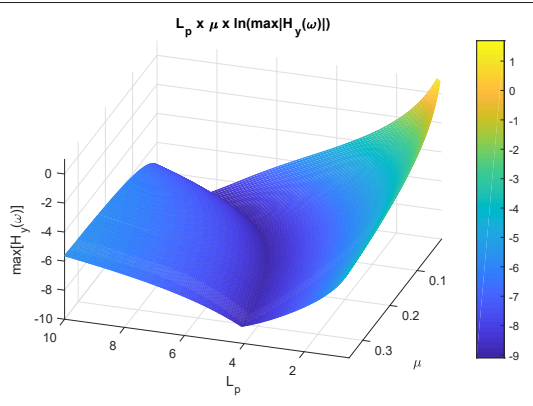

Figure 3. FEM BEAM+PTMD Response map - 3D view

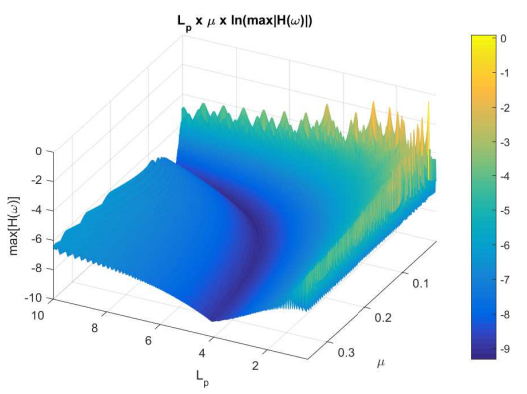

Figure 4. Analytical 2DOF Tower+PTMD Response map - 3D view [13]

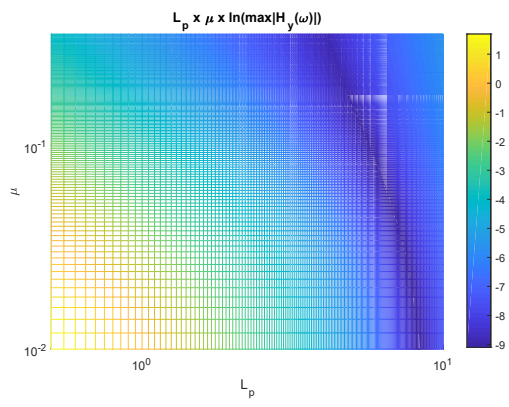

Figure 5. FEM BEAM+PTMD Response map - XY plan view

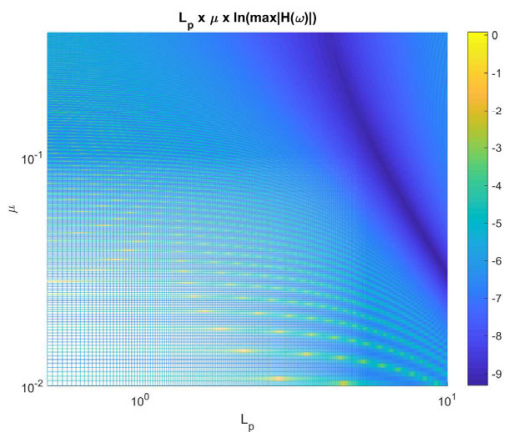

Figure 6. Analytical 2DOF Tower+PTMD Response map - XY plan view [13] 
see that when mass ratio is lower than $\mu>0.10$, the Analytical 2DOF Tower+PTMD represents well the FEM BEAM+PTMD system. Otherwise, for values of pendulum length over than $L_{p}>8,75 \mathrm{~m}$, there are any benefits for the PTMD controller.

\section{References}

[1] M.A. Lackner, M.A. Rotea, Wind Energy 14, 373 (2011)

[2] E.M. He, Y.Q. Hu, Y. Zhang, Proceedings of the Institution of Mechanical Engineers, Part M: Journal of Engineering for the Maritime Environment 231, 302 (2017)

[3] A. Deraemaeker, P. Soltani, Proceedings of the Institution of Mechanical Engineers, Part K: Journal of Multi-body Dynamics 231, 285 (2017)

[4] P. Xiang, A. Nishitani, Journal of Vibration and Control 23, 3128 (2017)

[5] T.T. Soong, G.F. Dargush, Journal of Structural Control 6, 172 (1999)

[6] S.M. Avila, M.A.M. Shzu, W.M. Pereira, L.S. Santos, M.V.G. Morais, Z.J.G. Prado, Journal of Vibration Engineering and Technologies 4(3), 249 - 257 (2016)

[7] M.A.M. Shzu, M.V.G. Morais, Z.J.G. del Prado, S.M. Ávila (2015)

[8] G.B. Colherinhas, M.A. Shzu, S.M. Avila, M.V. de Morais, Procedia Engineering 199, 1623 (2017)

[9] P. Murtagh, B. Basu, B. Broderick, Computers \& Structures 82, 1745 (2004)

[10] S. Adhikari, S. Bhattacharya, Shock and vibration 19, 37 (2012)

[11] Y. Ishida, T. Inoue, K. Nakamura, Journal of Environment and Engineering 4, 443 (2009)

[12] C. Felippa, University of Colorado at Boulder (2012)

[13] G.B. Colherinhas, M.V.G. Morais, M.A.M. Shzu, S.M. Ávila, CILAMCE 2016, Brasília, DF - XXXVII Ibero-Latin American Confress on Computational Methods in Engineering (2016) 